\title{
Physical activity does not alter prolactin levels in post-menopausal women: results from a dose-response randomized controlled trial
}

Darren R. Brenner ${ }^{1,2,3^{*}}$, Yibing Ruan ${ }^{1}$, Andria R. Morielli ${ }^{4}$, Kerry S. Courneya ${ }^{4}$ and Christine M. Friedenreich ${ }^{1,2,3}$

\begin{abstract}
Background: Increased circulating levels of prolactin have been associated with increased risk of both in situ and invasive breast cancer. We investigated whether or not physical activity had a dose-response effect in lowering plasma levels of prolactin in postmenopausal women.

Methods: Four hundred previously inactive but healthy postmenopausal women aged 50-74 years of age were randomized to 150 or 300 min per week of aerobic physical activity in a year-long intervention. Prolactin was measured from fasting samples with a custom-plex multiplex assay.

Results: A high compared to moderate volume of physical activity did not reduce plasma prolactin levels in intention-to-treat (Treatment Effect Ratio (TER) 1.00, 95\% Confidence Interval (CI) 0.95 - 1.06) or per-protocol analyses (TER 1.02, 95\% Cl $0.93-1.13$ ).

Conclusions: It is unlikely that changes in prolactin levels mediate the reduced risk of breast cancer development in post-menopausal women associated with increased levels of physical activity.
\end{abstract}

Trial registration: clinicaltrials.gov identifier: NCT01435005.

Keywords: Physical Activity, Randomized Controlled Trial, Breast Cancer, Prolactin

\section{Introduction}

The primary hypothesized mechanisms underlying the associations between physical activity and reduced breast cancer include reductions in adiposity, sex hormone levels, insulin resistance and chronic inflammation [1]. These pathways only explain some portion of the association and additional mechanisms require identification and further investigation.

Prolactin is a luteotropic peptide hormone involved in regular lactation which is produced by the anterior pituitary gland. Increased circulating levels of prolactin have been associated with increased risk of both in situ and invasive

\footnotetext{
* Correspondence: Darren.Brenner@albertahealthservices.ca

${ }^{1}$ Department of Cancer Epidemiology and Prevention Research,

CancerControl Alberta, Alberta Health Services, Holy Cross Centre, Room

513C, Box ACB, 2210 - 2nd St. SW, Calgary, AB T2S 3C3, Canada

${ }^{2}$ Department of Oncology, Cumming School of Medicine University of

Calgary, Calgary, AB, Canada

Full list of author information is available at the end of the article
}

breast cancer [2]. Two previous exercise intervention trials in post-menopausal women did not observe changes in prolactin levels in response to moderate physical activity $[3,4]$ compared to controls. As part of the Breast cancer and Exercise Trial in Alberta (BETA) we investigated: 1) the effects of increased levels of moderate to vigorous physical activity (MVPA) on levels of prolactin and 2) whether a higher level of activity led to larger changes in prolactin levels.

\section{Materials and methods}

The design of the BETA study has been previously described in detail [5]. Briefly, 400 previously inactive but otherwise healthy postmenopausal women of age were randomized to 150 (MODERATE) or 300 (HIGH) minutes per week of aerobic physical activity for a year-long intervention. Women were eligible for randomization if they were between 50-74 years of age, with no previous diagnosis of invasive cancer, no major comorbidities, 
obtained physical approval for participation, had a body mass index of 22-40, were moderately sedentary, not a current smoker or excessive drinker, not currently on a weight loss program, English speaking and not planning to be out of the study site areas for more than 4 consecutive weeks during the subsequent 18 months. Both intervention arms were prescribed the same frequency (five days/week) and intensity (moderate-to-vigorous) of aerobic exercise. The training targets were 60 minutes/session (300 total minutes/week) for the HIGH group, and 30 minutes/session (150 total minutes/week) for the MODERATE group. Wrist-worn heart rate were given to each participant and used to ensure a $65-75 \%$ maximum heart rate reserve (HRR) was being achieved during each exercise session. A ramp-up period was included where the intensity, frequency and duration of exercise were gradually increased during the first three months of the intervention until the target exercise prescriptions were attained. Fasting blood samples were collected from all participants at baseline, 6 and 12 months following a $24 \mathrm{~h}$ abstinence from alcohol intake and exercise and at least $10 \mathrm{~h}$ after their last meal. Prolactin levels in plasma were measured with a customplex multiplex assay (Eve Technologies, Calgary, AB, Canada), using the Bio-Plex ${ }^{\mathrm{mm}} 200$ system (Bio-Rad Laboratories, Inc., Hercules, CA, USA). The assay sensitivity for prolactin was $30.2 \mathrm{pg} / \mathrm{ml}$ and the inter-assay coefficient of variation was $5.5 \%$. Samples for each participant at different time points were all analyzed in the same batch and each batch included an equal number of MODERATE and HIGH blood samples.

Predicted $\mathrm{VO}_{2}$ max was estimated from a modified Balke treadmill test [6] using the multistage model and the American College of Sports Medicine metabolic equations for estimating maximum oxygen consumption [7].
Body composition was estimated from full body dual energy X-ray absorptiometry (DXA) scans to assess overall percent body fat and total fat mass. Computed tomography (CT) scans were taken at the level of the umbilicus to measure subcutaneous and intra-abdominal adiposity.

We considered the comparison of means of 12-month outcomes (log transformed, with no adjustment for baseline values) for the prolactin outcomes. Standard deviation was estimated from previous reports [8]. A sample size of 200 participants per group provided $90 \%$ power to detect anticipated changes of $4 \%$ between the treatment groups at $\alpha=0.05$. Prolactin changes in the two arms were compared in both intention-to-treat and per-protocol analysis using linear mixed models as previously described, adjusting for baseline prolactin levels [9]. A per-protocol analysis was conducted on participants achieving $\geq 60 \%$ of prescribed exercise duration in their target heart rate zone. To investigate whether the effects of exercise were restricted to particular subgroups, stratified analyses were conducted on a priori variables including baseline body mass index (BMI (weight $(\mathrm{kg}) /$ height $\left.\left(\mathrm{m}^{2}\right)\right)$, estimated physical fitness $\left(\mathrm{VO}_{2 \max }\right)$, and total percent body fat.

The study protocol was approved by the Alberta Cancer Research Ethics Committee and the Conjoint Health Research Ethics Board of the University of Calgary and the Health Research Ethics Board of the University of Alberta. All participants provided written informed consent.

\section{Results}

The distribution of the study participants' baseline characteristics was similar in the two trial arms with no meaningful differences between arms [9]. Baseline prolactin levels were $10565(\mathrm{SD}=6963) \mathrm{pg} / \mathrm{ml}$ in MODERATE

Table 1 Intention-to-treat and per-protocol analyses of prolactin concentrations between high and moderate volume exercise groups in BETA, $(n=386)$

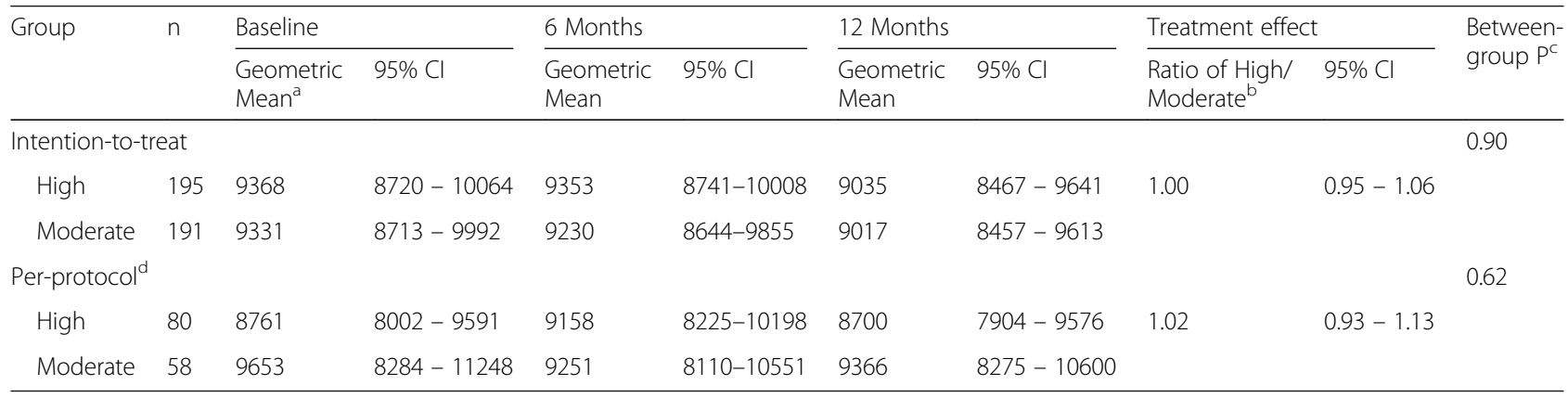

\footnotetext{
${ }^{\mathrm{a}}$ Geometric mean of prolactin was in the unit of $\mathrm{pg} / \mathrm{mL}$
}

${ }^{\mathrm{b}}$ The geometric mean ratios were estimated from least square means for the difference in treatment effect between high and moderate volume exercisers averaged across the entire study period adjusted for the baseline values and then back log-transformed

${ }^{c} P$ value corresponds to the null hypothesis that the ratio of treatment effect between high- and moderate-volume groups equals 1 against the 2 -sided alternative hypothesis

dWomen assigned to the moderate-volume group were adherent if they completed $90 \%$ to $100 \%$ of the exercise prescription (mean, $135-150$ min/week), weeks 13 to 52 at full prescription; women assigned to the high-volume group were adherent if they completed at least $90 \%$ of the exercise prescription (mean, $\geq 270 \mathrm{~min} /$ week), weeks 13 to 52 at full prescription 


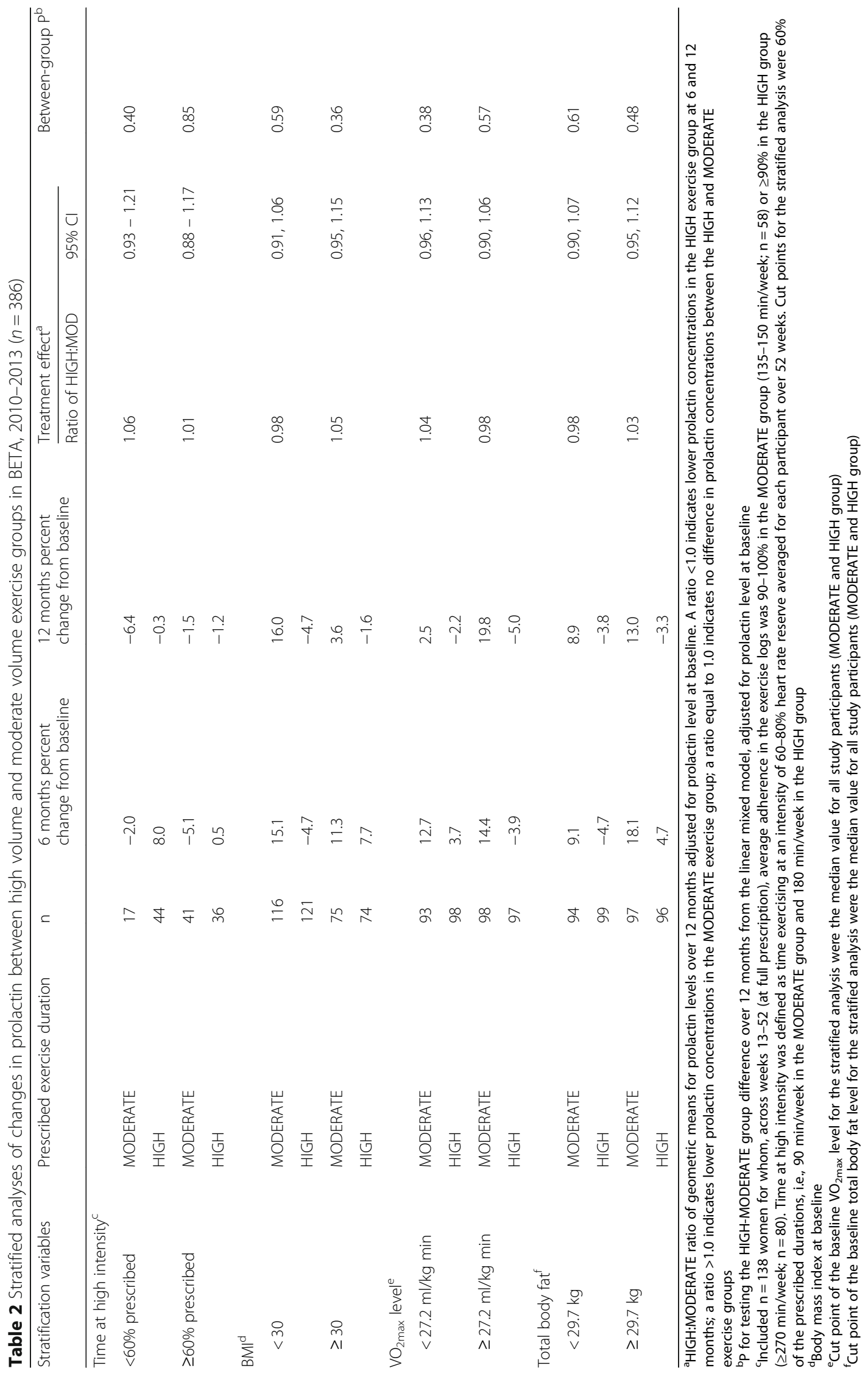


group and $10478(\mathrm{SD}=5150) \mathrm{pg} / \mathrm{ml}$ in HIGH group $(p=0.89)$. Overall, we did not observe statistically significant effects for increasing quartiles of physical activity and change in level of prolactin in the $n=384$ women included in the analyses (non-randomized analysis - results not shown).

No statistically significant differences were observed in the treatment effect ratios of the two exercise groups in either intention-to-treat (TER 1.00, 95\% CI 0.95 - 1.06) or per-protocol analyses (TER 1.02, 95\% CI 0.93 - 1.13; Table 1). A per-protocol analysis examining the role of exercise intensity ( $<$ or $\geq 60 \%$ of prescribed exercise) also showed no change in prolactin levels between the two groups (Table 1). In stratified analyses by estimated physical fitness $\left(\mathrm{VO}_{2 \max }\right)$ and total body fat, no treatment effect ratios in any of the subgroups were significantly different from 1.0 (Table 2).

\section{Discussion}

Overall, a higher volume of exercise compared to a standard volume did not reduce prolactin levels in BETA. Moreover, the effects did not vary according to exercise adherence or baseline fitness levels, BMI, and total body fat.

This exercise intervention trial is the first study to examine the effects of a high versus moderate volume of MVPA aerobic exercise on prolactin levels in postmenopausal women. Previous studies comparing 12 months of moderate-intensity aerobic exercise to no exercise have reported null effects on prolactin levels [3, 4]. Similarly, the results from these previous trials remained unchanged when exercise adherence, measured as minutes of exercise per day, was considered [3, 4]. In the study by Reding et al. [4], baseline BMI did not alter the treatment effect. In the study by Tworoger et al. [3], change in percent body fat did not mediate the intervention effect however, women in the exercise group who increased their $\mathrm{VO}_{2}$ max by $>5 \%$ had a statistically significant reduction in prolactin levels.

Our analyses were motivated by the strong animal and in vitro data supporting an important role of prolactin in breast carcinogenesis [10] and epidemiologic data suggesting an association of increased levels with breast cancer risk [11]. While our study did not observe significant impacts of exercise on levels of prolactin, it is worth noting that there are complex relationships between exercise and prolactin levels which may explain our null findings. For example, threshold effects of exercise intensity have been observed in the literature [12], which suggests that the intensity of exercise in the BETA trial may not have been high enough despite a target $>65 \%$ HRR. Furthermore, effects may differ by age as there are several studies among young athletic populations where increased levels of prolactin are reported post-acute bouts of exercise [12-14], while effects among older sedentary populations are less well characterized.

In conclusion, the results from the BETA study suggest that 300 min per week of moderate-intensity aerobic exercise does not reduce prolactin levels more than 150 min per week in a year-long intervention in postmenopausal women. It is unlikely that changes in prolactin levels mediate the reduced risk of breast cancer development in post-menopausal women associated with increased physical activity at any level.

\section{Acknowledgements \\ Calgary Study Coordinators were: Krista Carlson, Sana Fakih, Megan Farris, Quinn Harris, Sarah MacLaughlin, Erica Roberts, and Kristen Simone. Edmonton Study Coordinators were: Natalie Ilkiw, Ciara Kallal, and Dr. Amy Speed Andrews. Assistance with information sessions was provided in Calgary by Drs. Brigid Lynch and Fabiola Aparicio-Ting. Calgary Exercise Trainers were: Carrie Anderson, Alia Bharwani, Shannon Brown, Ashley Cuthbert, Sue Daniel, Julie Gowans, Margo Graham, Erin Korsbrek, Kathleen Kranenburg, Jessica Morrison, Jason Ng, Nicole Slot, Tania White, and Kaila Wright. Edmonton Exercise Trainers were: Arne Anderson, Lisa Belanger, Jennifer Crawford, Cindy Forbes, Alyssa Hindle, Corey Kuzik, Erin McGowan, Mary Norris, Janel Park, Julianne Symons, Linda Trinh, Stephanie Voaklander and Lynne Wong. Study recruiters were: Jennie Duke, Jasdeep Hayer, Trisha Kelly, Jasmine Lee, and Lilly Mah. Data entry was done by: Sinead Boyle, Barbara Mercer, Carla Quesnel and Trisha Kelly. Data management, including database creation, questionnaire design, data integrity and quality control, was done by: Dr. Steven Szarka, Farit Vakhetov, and Wendy Walroth. Qinggang Wang was responsible for the randomization procedures, sample size calculations and some data verification. The late Dr. Robert C. Millikan was a co-investigator on this trial and contributed to the study design and methods.}

\section{Funding}

This work was supported by a Career Development Award in Prevention (\#703917) from the Canadian Cancer Society Research Institute held by Dr. Brenner. Dr. Friedenreich holds a Health Senior Scholar Award from Alberta Innovates-Health Solutions and is the Alberta Cancer Foundation Weekend to End Women's Cancers Breast Cancer Chair. Dr. Courneya holds a Canada Research Chair in Physical Activity and Cancer.

\section{Availability of data and materials}

The datasets generated during and/or analyzed during the current study are not publicly available but are available from the corresponding author on reasonable request.

\section{Authors' contributions}

DB assisted in data collection, interpretation and wrote the manuscript. YR completed the data analysis and assisted in interpretation and writing of the manuscript. AM assisted in interpretation and writing of the manuscript. CF and KC were responsible for the design and conduct of the study, data collection as well as writing and critical review of the manuscript. All authors read and approved the final manuscript.

Ethics approval and consent to participate

This study was approved by the Health Research Ethics Board of Alberta and all participants consented to participate.

\section{Consent for publication}

Not applicable.

\section{Competing interests}

The authors declare that they have no competing interests.

\section{Publisher's Note}

Springer Nature remains neutral with regard to jurisdictional claims in published maps and institutional affiliations. 


\section{Author details}

'Department of Cancer Epidemiology and Prevention Research

CancerControl Alberta, Alberta Health Services, Holy Cross Centre, Room

513C, Box ACB, 2210 - 2nd St. SW, Calgary, AB T2S 3C3, Canada. ${ }^{2}$ Department of Oncology, Cumming School of Medicine University of Calgary, Calgary, AB, Canada. ${ }^{3}$ Department of Community Health Sciences, Cumming School of Medicine University of Calgary, Calgary, AB, Canada. ${ }^{4}$ Faculty of Physical Education and Recreation University of Alberta, Edmonton, AB, Canada.

Received: 28 December 2016 Accepted: 4 July 2017

\section{Published online: 13 July 2017}

\section{References}

1. Friedenreich CM, Neilson HK, Lynch BM. State of the epidemiological evidence on physical activity and cancer prevention. Eur J Cancer. 2010; 46(14):2593-604

2. Wang $M$, Wu X, Chai F, Zhang Y, Jiang J. Plasma prolactin and breast cancer risk: a meta- analysis. Sci Rep. 2016;6:25998.

3. Tworoger SS, Sorensen B, Chubak J, et al. Effect of a 12-month randomized clinical trial of exercise on serum prolactin concentrations in postmenopausa women. Cancer Epidemiol Biomarkers Prev. 2007;16(5):895-9.

4. Reding KW, Lampe JW, Wang CY, et al. A 12-month moderate-intensity exercise intervention does not alter serum prolactin concentrations. Cancer Epidemiol. 2011:35(6):569-73.

5. Friedenreich CM, MacLaughlin S, Neilson HK, et al. Study design and methods for the Breast Cancer and Exercise Trial in Alberta (BETA). BMC Cancer. 2014;14:919.

6. Pollock ML, Foster C, Schmidt D, Hellman C, Linnerud AC, Ward A. Comparative analysis of physiologic responses to three different maximal graded exercise test protocols in healthy women. Am Heart J. 1982;103(3):363-73.

7. Thompson PD, Arena R, Riebe D, Pescatello LS. American College of Sports M. ACSM's new preparticipation health screening recommendations from ACSM's guidelines for exercise testing and prescription, ninth edition. Curr Sports Med Rep. 2013;12(4):215-7.

8. Tanner MJ, Hadlow NC, Wardrop R. Variation of female prolactin levels with menopausal status and phase of menstrual cycle. Aust N Z J Obstet Gynaecol. 2011:51(4):321-4.

9. Friedenreich CM, Neilson HK, O'Reilly R, et al. Effects of a High vs Moderate Volume of Aerobic Exercise on Adiposity Outcomes in Postmenopausal Women: A Randomized Clinical Trial. JAMA Oncol. 2015;1(6):766-76.

10. Clevenger CV, Furth PA, Hankinson SE, Schuler LA. The role of prolactin in mammary carcinoma. Endocr Rev. 2003;24(1):1-27.

11. Tworoger SS, Eliassen AH, Sluss P, Hankinson SE. A prospective study of plasma prolactin concentrations and risk of premenopausal and postmenopausal breast cancer. J Clin Oncol. 2007;25(12):1482-8.

12. Chang FE, Dodds WG, Sullivan M, Kim MH, Malarkey WB. The acute effects of exercise on prolactin and growth hormone secretion: comparison between sedentary women and women runners with normal and abnormal menstrual cycles. J Clin Endocrinol Metab. 1986;62(3):551-6.

13. De Cree C. Sex steroid metabolism and menstrual irregularities in the exercising female. A review Sports Med. 1998;25(6):369-406.

14. Boyden TW, Pamenter RW, Grosso D, Stanforth P, Rotkis T, Wilmore JH. Prolactin responses, menstrual cycles, and body composition of women runners. J Clin Endocrinol Metab. 1982;54(4):711-4.

\section{Submit your next manuscript to BioMed Central and we will help you at every step:}

- We accept pre-submission inquiries

- Our selector tool helps you to find the most relevant journal

- We provide round the clock customer support

- Convenient online submission

- Thorough peer review

- Inclusion in PubMed and all major indexing services

- Maximum visibility for your research

Submit your manuscript at www.biomedcentral.com/submit
Biomed Central 